\title{
Calcifying uterine leiomyoma invading the urinary bladder
}

\author{
G. Hudelist • A. Fouad • J. English • T. Liston • R. Beard
}

Received: 14 January 2007 / Accepted: 28 April 2007 / Published online: 1 June 2007

(C) Springer-Verlag 2007

\begin{abstract}
Leiomyomas are common benign tumors of the female genital tract and can be the leading cause of dysfunctional uterine bleeding, recurrent miscarriage or abdominal discomfort. Degeneration of fibroids occurs in menopause and is associated with fatty, hyaline, cystic, or myxoid degeneration or dystrophic calcification. However, based on their benign nature, these tumors are not known to invade other tissues. We here describe a rare case of an extensively calcified myoma invading the urinary bladder in an elderly patient who presented with hematuria and moderate abdominal discomfort.
\end{abstract}

Keywords Uterine leiomyoma $\cdot$ Calcification .

Bladder invasion

\section{Introduction}

Benign neoplasms of the uterine muscle are common entities and can be found in up to $30 \%$ of the reproductive female population [1]. Leiomyomas most commonly involve the uterine fundus and corpus but can also be

\footnotetext{
G. Hudelist $(\square) \cdot$ A. Fouad $\cdot$ J. English

Department of Obstetrics and Gynaecology,

Worthing and Southlands Hospital,

Lyndhurst Road,

BN11 2DH Worthing, West Sussex, UK

e-mail: gernot_hudelist@yahoo.de

G. Hudelist

Department of Obstetrics and Gynaecology LKH,

Villach, Austria

T. Liston $\cdot$ R. Beard

Department of Urology, Worthing and Southlands Hospital,

Worthing, UK
}

observed in the cervix or round ligaments and are composed of smooth muscle and varying amounts of fibrous connective tissue. Submucous growth and distortion of the endometrial cavity can be associated with dysfunctional uterine bleeding and subfertility due to impaired implantation [2]. On rare occasions, abdominal discomfort or even pelvic pain is caused by gross enlargment of these tumors. Although leiomyomas have been reported to reach sizes of up to $30 \mathrm{~cm}$ in diameter [3] and weights of over $40 \mathrm{~kg}$ [4], these tumors do not usually penetrate surrounding tissues due to their benign nature and encapsulated condition. We here report a case of a calcifying uterine fibroid with bladder invasion associated with hematuria as the primary clinical manifestation of disease.

\section{Case report}

A 68-year-old (gravida 0 para 0 ) female of Caucasian origin presented with dysuria, urinary frequency and moderate abdominal discomfort. She had a known history of chronic hypertension and arthritis. Cervical smears had been normal. Her gynecological history did not reveal any abnormalities, and she experienced menopause at the age of 52. At presentation in September 2006, urinalysis revealed microhematuria and moderate leukocyturia lacking significant growth of organisms on urine culture. An ultrasound scan of her kidneys revealed a cystic structure of $3 \times 3 \times 3.3 \mathrm{~cm}$ in the upper pole of her left kidney suggesting a simple kidney cyst but was otherwise normal. Flexible cystoscopy for further evaluation of her hematuria revealed three large solid structures corresponding to bladder stones eroding the urothelium. On clinical examination, a hard, nodular but movable mass was palpated in her lower abdomen with suprapubic extension up to the 


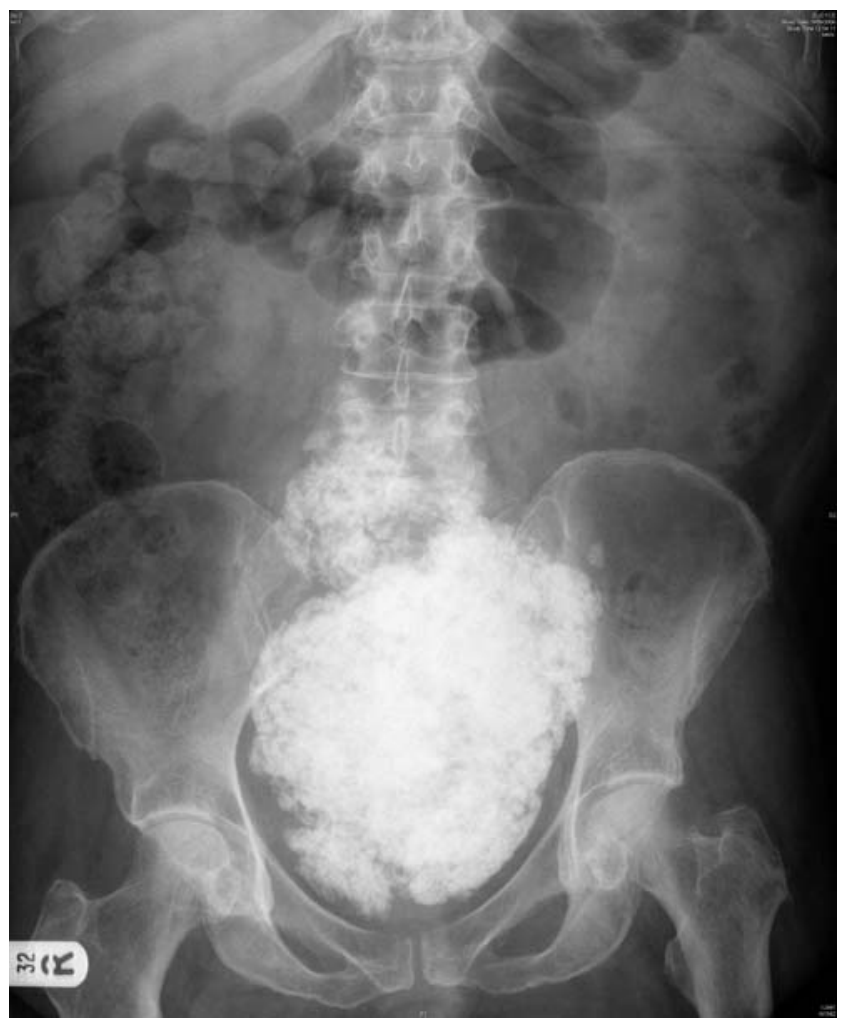

Fig. 1 Plain abdominal X-ray exhibiting multiple calcifications within an unclearly defineable mass arising out of the pelvis

level of the umbilicus. An X-ray of the abdomen showed multiple calcifications within an unclearly defined mass extending out of the pelvis (Fig. 1). Computer tomography (CT) of the abdomen and pelvis revealed a nonhomogeneous $10 \times 15 \times 12 \mathrm{~cm}$ solid mass of unknown origin and questionable communication with the bladder lumen but otherwise lacking signs of infiltration into surrounding pelvic structures. Finally, an MRI of the pelvis was carried out again showing a $15 \times 15 \times 12 \mathrm{~cm}$ large mixed-signal mass arising from the anterior aspect of the uterus extending up to the aortic bifurcation. High-signal urine was seen to extend into this area suggesting communication with the bladder (Fig. 2).

At operation, the tumor presented as a solid mass originating from the uterine corpus. There was no sign of peritoneal metastasis or ascites or peritoneal fluid in the pouch of Douglas. However, the bladder was found to be densely adhering to the anterior wall of the structure and could not be dissected from the cervix. Incision of the bladder lumen revealed multiple calcifications eroding the posterior bladder wall. The patient then underwent subtotal abdominal hysterectomy (i.e. preservation of the cervix), bilateral salpingo-oophorectomy and partial resection of the infiltrated posterior wall of the urinary bladder (Fig. 3). Histological analysis of paraffin-embedded tissue confirmed a benign smooth muscle tumor with extensive

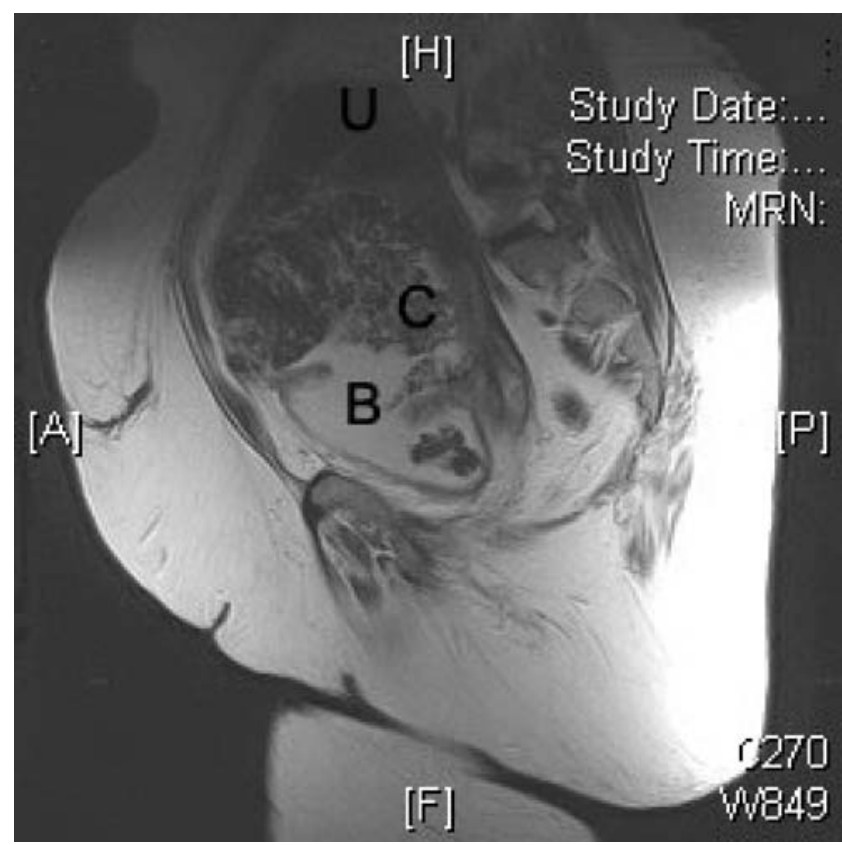

Fig. 2 MRI of the pelvis demonstrating a $15 \times 15 \times 12 \mathrm{~cm}$ large mixedsignal mass arising from the anterior aspect of the uterus. Signal-intense urine appears to extend into this area suggesting communication with the bladder. $U$ Uterus, $B$ bladder, $C$ calcifications

calcifications, which continuously replaced the majority of the smooth muscle layers of the posterior wall of the bladder where calcified tissue was observed to protrude into the bladder wall macroscopically. Her post-operative course was uncomplicated, and the urinary catheter was removed on day 9 after the operation without complication. The patient was finally discharged following a control cystogram to exclude leakage. At follow-up 4 weeks postoperatively, the patient was complaining of moderate symptoms of urge incontinence but was otherwise doing well. A bladder scan showed minimal residual volume.

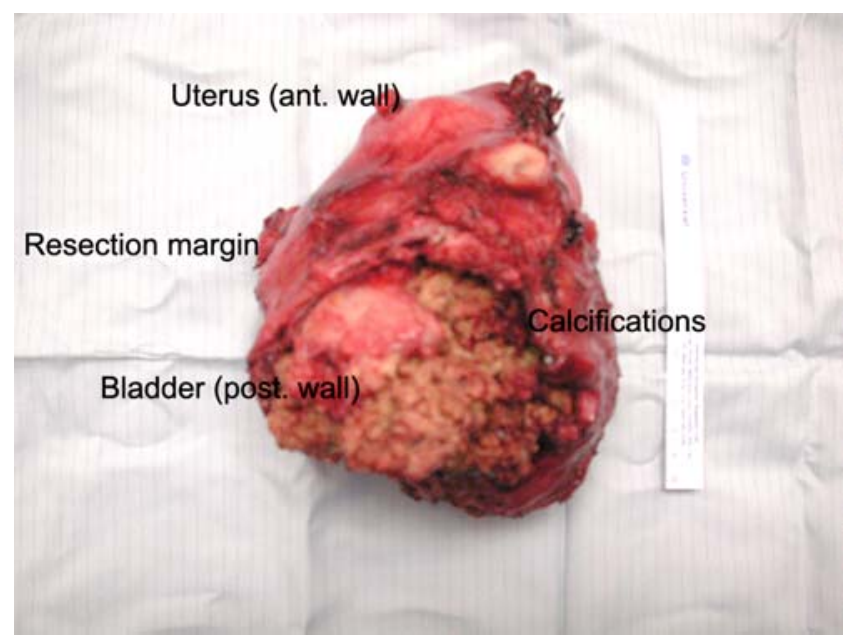

Fig. 3 Large uterine leiomyoma with extensive calcifications eroding the posterior wall of the urinary bladder 


\section{Discussion}

Various types of degeneration of leiomyomatous tissue have been reported as a consequence of gross enlargement and a relative reduction in sufficient blood supply. These include hyaline, cystic, myxoid or dystrophic calcifications [5]. Whereas hyalinization can be observed in up to $60 \%$ of cases, cystic degeneration and calcification are less common and occur in about 5\% [6]. Calcification of fibroids usually appears dense and amorphous on plain radiography or may exhibit ring-like structures corresponding to thrombosed veins from previous stages of degeneration undergoing calcification [6, 7].

Although uterine fibroids may greatly enlarge and deviate the position of surrounding organs such as the bladder, their growth pattern is expansive and usually follows rounded borders in contrast to diffuse and infiltrative growth of sarcomatous tissue. The case presented in this report emerges as a rare exception of this rule by exhibiting invasion into the posterior wall of the bladder despite its entirely benign nature. One possible explanation for this rather unusual growth pattern is the presence of multiple stone-like structures representing extensive calcification of leiomyomatous tissue. We suggest that mechanical irritation of mucosal and muscular tissue layers of the bladder was followed by secondary atrophy and erosion of the posterior wall during further enlargement of the fibroid. This was associated with hematuria and urinary frequency. Although uterine leiomyomata have been shown to display a wide spectrum of appearances on radiological imaging studies reflected by either ill-defined borders or varying degrees of echogenity or signal intensity, we here report a case exhibiting infiltrative rather than expansive growth of these benign tumors thereby adding to the diagnostic confusion.

\section{References}

1. Ouyang DW, Economy KE, Norwitz ER (2006) Obstetric complications of fibroids. Obstet Gynecol Clin North Am 33:153-169

2. Casini ML, Rossi F, Agostini R, Unfer V (2006) Effects of the position of fibroids on fertility. Gynecol Endocrinol 22:106-109

3. Grapsa D, Smymiotis V, Hasiakos D, Kontogianni-Katsarou K, Kondi-Pafiti A (2006) A giant uterine leiomyoma simulating an ovarian mass in a 16-year-old girl: a case report and review of the literature. Eur J Gynaecol Oncol 27:294-296

4. Oelsner G, Elizur SE, Frenkel Y, Carp H (2003) Giant uterine tumors: two cases with differential presentations. Obstet Gynecol 101:1088-1091

5. Prayson RA, Hart WR (1995) Pathologic considerations of uterine smooth muscle tumors. Obstet Gynecol Clin North Am 22:637-657

6. Ueda H, Togashi K, Konishi I, Kataoka ML, Koyama T, Fujiwara T, Kobayashi H, Fujii S, Konishi J (1999) Unusual appearances of uterine leiomyomas: MR imaging findings and their histopathological backgrounds. Radiographics 19:131-145

7. Mayer DP, Shipilov V (1995) Ultrasonography and magnetic resonance imaging of uterine fibroids. Obstet Gynecol Clin North Am 22:667-725 